\title{
DECOMPOSITION THEORY FOR NONSEMIMODULAR LATTICES
}

\author{
BY \\ PETER CRAWLEY(')
}

1. Introduction. In a previous paper [1] a theory of meet decompositions in compactly generated atomic lattices was developed which for the most part generalized the classical theory for finite dimensional lattices. It was shown that if a compactly generated atomic lattice is semimodular, then every element has an irredundant meet decomposition into completely irreducible elements. Every element of a compactly generated atomic lattice has a unique irredundant decomposition if and only if the lattice is locally distributive. Aside from uniqueness the most fundamental arithmetical property of irredundant decompositions is the Kurosh-Ore replacement property, that is, for every pair of irredundant decompositions $a=\cap Q=\cap Q^{\prime}$ of an element $a$ and for each irreducible $q \in Q$ there is an irreducible $q^{\prime} \in Q^{\prime}$ such that $a=q^{\prime}$ $\cap \cap(Q-q)$. Every modular lattice has this property. Moreover, it was shown that a semimodular, compactly generated, atomic lattice has the replacement property if and only if the lattice is locally modular.

All of these results used heavily the semimodular condition. Only the characterization of unique decompositions was carried out for general lattices, and this was accomplished because semimodularity could be proved in this case.

The present paper extends the decomposition theory to include nonsemimodular lattices. It is shown in the second section that the elements of any compactly generated atomic lattice have irredundant decompositions. In the third section a necessary and sufficient condition is obtained for an arbitrary compactly generated atomic lattice to have the replacement property. This condition is a modification of the lower-semimodular law, and in the presence of semimodularity is easily seen to be equivalent to local modularity.

Throughout this paper the notation and terminology of [1] is used. Lattice join, meet, inclusion, proper inclusion, and covering, are denoted respectively by the symbols $\cup, \cap, \leqq,<$, and $\prec$. The corresponding set operations are denoted respectively by the symbols $\vee, \wedge, \subseteq$, and $\subset$. If $S$ and $T$ are sets, then $S-T=\{x \mid x \in S, x \in T\} ;$ if $T$ contains a single element $t$, then $S-T$ is also written $S-t$. If $a, b$ are elements of a lattice $L$ with $b \geqq a$, then the quotient sublattice $b / a$ is defined by $b / a=\{x \in L \mid a \leqq x \leqq b\}$. A lattice $L$ is atomic if whenever $b>a$ in $L$ there is an element $p \in L$ with $b \geqq p>a$. In a

Received by the editors November 7,1960 .

(1) This work was supported in part by the Office of Naval Research. 
complete atomic lattice $L$ the element $u_{a}$ is defined for each $a \in L$ by $u_{a}$ $=U\{p \mid p>a\}$. An element $c$ in a complete lattice $L$ is compact if for each subset $S \subseteq L$ with $c \leqq U S$ there is a finite subset $S^{\prime} \subseteq S$ such that $c \leqq \cup S^{\prime}$. A lattice $L$ is called compactly generated if $L$ is complete and each element of $L$ is a join of compact elements. A lattice $L$ is semimodular if $x>x \cap y$ implies $x \cup y>y$ for all $x, y \in L$. $L$ is lower-semimodular if $x \cup y>x$ implies $y>x \cap y$. A complete atomic lattice is locally modular if the sublattice $u_{a} / a$ is modular for each $a \in L$. Local distributivity is analogously defined. An element $q$ in a complete lattice $L$ is completely irreducible if for each $S \subseteq L, q=\cap S$ implies $q \in S$. A representation of an element as a meet of completely irreducible elements is said to be a decomposition of the element. A decomposition $a=\cap Q$ of an element $a$ is irredundant if $\cap(Q-q) \neq a$ for each $q \in Q$.

2. The existence of irredundant decompositions. This section contains a proof that irredundant decompositions exist in any compactly generated atomic lattice. We begin with the following four lemmas.

LEMMA 2.1. If $a, b$ are elements of a compactly generated lattice $L$ and $b>a$, then the quotient sublattice $b / a$ is also compactly generated.

LEMMA 2.2. If $a$ is an element of a compactly generated lattice $L$ and $\left\{x_{\alpha}\right\}$ is a chain of $L$, then $a \cap \bigcup_{\alpha} x_{\alpha}=\cup_{\alpha} a \cap x_{\alpha}$.

LEMмA 2.3. If $a, b$ are elements of a compactly generated lattice $L$ and $a \geq b$, then there exists a completely irreducible element $q \in L$ such that $q \geqq a$ and $q \geqq b$.

LEMMA 2.4. If $a, b$ are elements of a compactly generated lattice $L$, then there exists a maximal element $m \in L$ such that $m \geqq a$ and $m \cap b=a \cap b$.

Lemmas 2.1, 2.2, and 2.3 are given respectively in [1, Lemmas 3.1, 2.3, and 2.5]. To show Lemma 2.4 let $T=\{x \in L \mid x \geqq a, x \cap b=a \cap b\} . T$ is then nonempty since $a \in T$. If $\left\{x_{\alpha}\right\}$ is a chain of $T$, then by Lemma 2.2 we have $b \cap \bigcup_{\alpha} x_{\alpha}=U_{\alpha} b \cap x_{\alpha}=a \cap b$, whence $U_{\alpha} x_{\alpha} \in T$. Thus by the Maximal Principle $T$ contains a maximal element $m$.

The existence theorem is now the following.

THEOREM 2.1. Every element of a compactly generated atomic lattice has an irredundant decomposition into completely irreducible elements.

Proof. Let $a$ be an element of the lattice distinct from the unit element. Then by atomicity there is an element $p$ with $p>a$. By Lemma 2.3 there exists a completely irreducible element $q$ such that $q \geqq a$ and $q \geqq p$, and hence $a=q \cap p$. Thus to prove the theorem it suffices to prove the following lemma.

LemMa 2.5. Let $L$ be a compactly generated lattice. Then every element of $L$ has an irredundant decomposition if and only if for each element $a \in L$ distinct from the unit element of $L$ there exist a completely irreducible element $q$ and an element $x>a$ such that $a=q \cap x$. 
The necessity is clear. To prove the sufficiency, let $a \in L$ be an element which is distinct from the unit element.

Let $W$ be the collection of all ordered pairs $(R, x)$ such that $R$ is a set of completely irreducible elements of $L, x \in L$, and such that the following conditions are satisfied:

(1) $x \cap \cap R=a$,

(2) $x \cap \cap(R-q)>a$ for all $q \in R$.

Partially order $W$ by defining $(R, x) \geqq\left(R^{\prime}, x^{\prime}\right)$ if and only if the following two conditions hold:

(3) $R \supseteq R^{\prime}$,

(4) $x \cap \cap\left(R-R^{\prime}\right) \geqq x^{\prime}$.

Now by the assumption of the lemma there exist an element $x_{0} \in L$ and a completely irreducible element $q_{0} \in L$ such that $x_{0}>a$ and $a=x_{0} \cap q_{0}$. Then with $R_{0}=\left\{q_{0}\right\}$, the ordered pair $\left(R_{0}, x_{0}\right)$ is a member of $W$, and hence $W$ is nonempty. By the Maximal Principle, $W$ contains a maximal chain $\left\{\left(R_{\alpha}, x_{\alpha}\right)\right\}$.

Define

(5) $Q=\mathrm{V}_{\alpha} R_{\alpha}$,

(6) $y=U_{\alpha} x_{\alpha}$.

Since $\left\{x_{\alpha}\right\}$ forms a chain by (4), it follows from Lemma 2.2 and condition (1) that

$$
a \leqq y \cap \cap Q=(\cap Q) \cap \bigcup_{\alpha} x_{\alpha}=\bigcup_{\alpha}\left(x_{\alpha} \cap \cap Q\right) \leqq \bigcup_{\alpha}\left(x_{\alpha} \cap \cap R_{\alpha}\right)=a
$$

whence $a=y \cap \cap Q$. Now let $q$ be any element of $Q$. Then for some index $\alpha$ we have $q \in R_{\alpha}$. By conditions (5), (3), and (4), if $q^{\prime}$ is any other element of $Q$, then either $q^{\prime} \in R_{\alpha}$ or $q^{\prime} \geqq x_{\alpha}$. Since clearly $y \geqq x_{\alpha}$, it follows that $y \cap \cap\left(Q-R_{\alpha}\right)$ $\geqq x_{\alpha}$. Thus by condition (2)

$$
y \cap \cap(Q-q)=\left[y \cap \cap\left(Q-R_{\alpha}\right)\right] \cap \cap\left(R_{\alpha}-q\right) \geqq x_{\alpha} \cap \cap\left(R_{\alpha}-q\right)>a .
$$

In particular, $\cap(Q-q)>a$ for every $q \in Q$.

Therefore to show that $a$ has an irredundant decomposition it suffices to show that $\cap Q=a$. Suppose $\cap Q>a$. Since $y \cap \cap Q=a$, by Lemma 2.4 there exists a maximal element $m \in L$ such that $m \geqq y$ and $m \cap \cap Q=a$. $m$ cannot be the unit element of $L$ since $\cap Q>a$. Hence by the assumption of the lemma there exist an element $t>m$ and a completely irreducible element $r \geqq m$ such that $t \cap r=m$. Let $Q_{1}=Q \bigvee\{r\}$. We then have

$$
t \cap \cap Q_{1}=t \cap r \cap \cap Q=m \cap \cap Q=a .
$$

If $q$ is any element of $Q$, then

$$
t \cap \cap\left(Q_{1}-q\right)=m \cap \cap(Q-q) \geqq y \cap \cap(Q-q)>a .
$$

Since $t>m$, the maximal property of $m$ implies that 


$$
t \cap \cap\left(Q_{1}-r\right)=t \cap \cap Q>a .
$$

Hence $\left(Q_{1}, t\right) \in W$. Furthermore, if $\left(R_{\alpha}, x_{\alpha}\right)$ is any element of the chain $\left\{\left(R_{\alpha}, x_{\alpha}\right)\right\}$, then $Q_{1} \supseteq Q \supseteq R_{\alpha}$, and

$$
t \cap \cap\left(Q_{1}-R_{\alpha}\right)=m \cap \cap\left(Q-R_{\alpha}\right) \geqq y \cap \cap\left(Q-R_{\alpha}\right) \geqq x_{\alpha} .
$$

And since $t>m \geqq y \geqq x_{\alpha}$, it follows that $\left(Q_{1}, t\right)>\left(R_{\alpha}, x_{\alpha}\right)$. This implies that $\left\{\left(R_{\alpha}, x_{\alpha}\right)\right\} \vee\left\{\left(Q_{1}, t\right)\right\}$ is a chain of $W$ properly containing the maximal chain $\left\{\left(R_{\alpha}, x_{\alpha}\right)\right\}$. Since this is inpossible, we must have $a=\bigcap Q$, completing the proof of the theorem.

In the proof of the preceding theorem the Axiom of Choice (in the form of the Maximal Principle) was used several times. It is perhaps interesting that conversely Theorem 2.1 implies the Axiom of Choice. For if $P$ is any partially ordered set and $L$ is the collection of all chains of $P$ together with the null set and $P$ itself, then it is easily seen that $L$ is a compactly generated atomic lattice under set inclusion. Moreover every completely irreducible element of $L$ not equal to $P$ is either a maximal chain of $P$ or covered by a maximal chain. Hence if completely irreducible elements not equal to $P$ exist in $L$, in particular if Theorem 2.1 holds, then $P$ has a maximal chain.

3. Lattices with replaceable decompositions. If $a$ is an element of a compactly generated atomic lattice $L$, we shall say that $a$ has replaceable irredundant decompositions if for every pair of irredundant decompositions $a=\cap Q=\cap Q^{\prime}$ and each $q \in Q$ there exists an element $q^{\prime} \in Q^{\prime}$ such that $a=q^{\prime} \cap \cap(Q-q)$, and this decomposition. is irredundant. The principal theorem of this section characterizes those lattices $L$ which have replaceable irredundant decompositions.

For every pair of elements $x, y \in L$ define the element $u_{x / y}$ by

$$
u_{x / y}=U\{p \mid p \leqq x, p>y\} .
$$

THEOREM 3.1. Every element of a compactly generated atomic lattrce $L$ has replaceable irredundant decompositions if and only if $L$ satisfies the following condition:

(o) If $x, y \in L$, then $u_{x \cup y / x}>x$ implies $u_{y / x \cap y}>x \cap y$.

REMARK. Condition ( $\rho$ ) may be stated alternatively as follows. If $x, y$ are any two elements of $L$ and the quotient sublattice $x \cup y / x$ contains a unique element covering $x$, then the sublattice $y / x \cap y$ contains a unique element covering $x \cap y$.

Proof of Theorem 3.1. The proof of the theorem is based on the following lemmas.

Lemma 3.1. An element $a \in L$ has replaceable irredundant decompositions if and only if $q \cap\left(p_{1} \cup p_{2}\right)=a$ implies $p_{1}=p_{2}$ for every completely irreducible element $q \geqq a$ and every pair of elements $p_{1}, p_{2}>a$. 
Let $a \in L$ be an element for which the condition of Lemma 3.1 is satisfied. Let $a=\cap Q=\cap Q^{\prime}$ be two irredundant decompositions of $a$, and let $q \in Q$. Since $a=q \cap \cap(Q-q)$, it follows from the condition of the lemma that there is a unique element $p>a$ such that $\cap(Q-q) \geqq p$. Because $\cap Q^{\prime}=a$, there is an element $q^{\prime} \in Q^{\prime}$ such that $q^{\prime} \geq p$, and hence it follows from the atomicity of $L$ that $q^{\prime} \cap \cap(Q-q)=a$. Suppose this decomposition is redundant. Then there exists an element $q_{1} \in Q-q$ such that $a=q^{\prime} \cap \cap\left(Q-\left\{q, q_{1}\right\}\right)$. Again since $q^{\prime} \cap \cap\left(Q-\left\{q, q_{1}\right\}\right)=a$ and $a$ satisfies the condition of the lemma, it follows that there is a unique element $p_{1}>a$ such that $\cap\left(Q-\left\{q, q_{1}\right\}\right) \geqq p_{1}$. But since $\cap\left(Q-\left\{q, q_{1}\right\}\right) \geqq \cap(Q-q) \geqq p$, we must have $p=p_{1}$. This implies that $q \cap \cap\left(Q-\left\{q, q_{1}\right\}\right)=\cap\left(Q-q_{1}\right)=a$, contrary to the irredundance of the decomposition $a=\cap Q$. Hence the decomposition $a=q^{\prime} \cap \cap(Q-q)$ is irredundant, and $a$ has replaceable irredundant decompositions.

Suppose now that $a \in L$ has replaceable irredundant decompositions. Let $q \geqq a$ be a completely irreducible element and $p_{1}, p_{2}>a$ be elements such that $a=q \cap\left(p_{1} \cup p_{2}\right)$. By Lemma 2.4 there exists a maximal element $m \geqq p_{1} \cup p_{2}$ such that $q \cap m=a$. Let $m=\cap R$ be an irredundant decomposition of $m$. Then because of the maximality of $m$ it follows that $a=q \cap \cap R$ is an irredundant decomposition of $a$. If $p_{1} \neq p_{2}$, then by Lemma 2.3 there exists a completely irreducible element $q_{1} \geqq p_{1}$ such that $q_{1} \geq p_{2}$, and hence $q_{1} \cap p_{2}=a$. Let $m_{1} \geqq p_{2}$ be a maximal element such that $q_{1} \cap m_{1}=a$, and let $m_{1}=\bigcap R_{1}$ be an irredundant decomposition of $m_{1}$. Then again it follows from the maximality of $m_{1}$ that $a=q_{1} \cap \cap R_{1}$ is an irredundant decomposition of $a$. But now it follows that $q_{1} \cap \cap R \geqq p_{1}>a$, and $q^{\prime} \cap \cap R \geqq p_{2}>a$ for every $q^{\prime} \in R_{1}$. Since this is contrary to the assumption that $a$ has replaceable irredundant decompositions we must have $p_{1}=p_{2}$, and hence the lemma follows.

LеммA 3.2. If an element $a \in L$ has replaceable irredundant decompositions in $L$, then a has replaceable irredundant decompositions in the sublattice $x / a$ for every $x>a$.

For suppose $r$ is a completely irreducible element of the quotient sublattice $x / a$ and $r \cap\left(p_{1} \cup p_{2}\right)=a$ for elements $p_{1}, p_{2}$ such that $x \geqq p_{1}, p_{2}>a$. If $r=\bigcap Q$ is a decomposition of $r$ into elements which are completely irreducible in $L$, then

$$
r=x \cap \cap Q=\bigcap_{q \in Q} x \cap q,
$$

and since $r$ is completely irreducible in $x / a$ it follows that $r=x \bigcap q$ for some irreducible $q \in Q$. Hence

$$
q \cap\left(p_{1} \cup p_{2}\right)=q \cap x \cap\left(p_{1} \cup p_{2}\right)=r \cap\left(p_{1} \cup p_{2}\right)=a,
$$

and since $a$ has replaceable irredundant decompositions in $L$ it follows from Lemma 3.1 that $p_{1}=p_{2}$. Thus, since $x / a$ is a compactly generated atomic lattice, $a$ has replaceable irredundant decompositions in the sublattice $x / a$. 
Proceeding now with the proof of the theorem, let $L$ be a compactly generated atomic lattice satisfying condition $(\rho)$. Let $a \in L, q \geqq a$ be an irreducible of $L$, and $p_{1}, p_{2}>a$ be elements such that $q \cap\left(p_{1} \cup p_{2}\right)=a$. Since $q$ is completely irreducible in $L$, there is a unique element $s$ covering $q$. Thus $u_{p_{1} \cup p_{2} \cup q / q}=s>q$, and hence by condition $(\rho)$ it follows that $u_{p_{1} \cup p_{2} / a}>a$. This implies that $p_{1}=p_{2}$, and hence by Lemma 3.1 every element of $L$ has replaceable irredundant decompositions.

Suppose every element o: $L$ has replaceable irredundant decompositions. Let $x, y \in L$ be such that $u_{x \cup y / x}>x$. If $u_{y / x \cap y}$ does not cover $x \cap y$, then there are two distinct elements $p_{1}, p_{2}$ such that $y \geqq p_{1}, p_{2}>x \cap y$. Since $u_{x \cup y / x}>x$ it follows that $x$ is completely irreducible in the quotient sublattice $x \cup y / x \cap y$. And since $x \cap\left(p_{1} \cup p_{2}\right)=x \cap y$ for two distinct elements $p_{1}, p_{2}>x \cap y$, it follows from Lemma 3.1 that $x \cap y$ does not have replaceable irredundant decompositions in the sublattice $x \cup y / x \cap y$. But then by Lemma 3.2 it follows that $x \cap y$ does not have replaceable decompositions in $L$. This contradiction implies that $u_{y / x \cap y}>x \cap y$. Thus $L$ satisfies $(\rho)$, and the proof of Theorem 3.1 is complete.

It is clear that if $L$ is a point lattice $\left({ }^{2}\right)$, that is, if $x$ is the join of elements covering $y$ for every pair of elements $x>y$ in $L$, then condition $(\rho)$ is equivalent to lower-semimodularity. Therefore a compactly generated point lattice has replaceable irredundant decompositions if and only if the lattice is lowersemimodular.

THEOREM 3.2. If $L$ is a semimodular, compactly generated, atomic lattice, then $L$ satisfies condition $(\rho)$ if and only if $L$ is locally modular.

Proof. If $L$ is locally modular, then $(\rho)$ follows immediately from [1, Lemma 7.1]. Suppose then that $L$ satisfies condition ( $\rho)$. If $a \in L$ and every element of $u_{a} / a$ is a join of elements covering $a$, then $u_{a} / a$ is a point lattice. For if $x>y$ in $u_{a} / a$, then $x=\cup\{p \cup y \mid p>a, p \leqq x, p \leq y\}$, and since $p \$ y$ implies $p \cup y>y$, the assertion follows. Hence, in view of [1, Lemma 3.4] and the remark following the proof of Theorem 3.1 above, to show that $L$ is locally modular it suffices to show that every element of $u_{a} / a$ is a join of elements covering $a$ for each $a \in L$. Since $u_{a} / a$ is compactly generated we need only show that each compact element is a join of elements covering $a$. If $c$ is a compact element of $u_{a} / a$, then there is a minimal finite number of elements $p_{1}, \cdots, p_{k}>a$ such that $p_{1} \cup \ldots \cup p_{k} \geqq c$. Since $L$ is semimodular, every chain of $p_{1} \cup \ldots \cup p_{k} / a$ is of length at most $k$. Thus, if $p_{1} \cup \ldots \cup p_{k}>c$ and $t$ is such that $p_{1} \cup \ldots \cup p_{k}>t \geqq c$, then $t$ is not a join of elements covering $a$. Let $t_{1}=\cup\{p>a \mid p \leqq t\}$. Then since $p_{1} \cup \cdots \cup p_{k} / t_{1}$ has dimension at least two, there are two distinct elements $p_{i}, p_{j}$ such that $t_{1} \geq p_{i}, p_{j}$, and $t_{1} \cup p_{i} \geq p_{j}$. Now $p_{i} \cup p_{j} \cup t=p_{1} \cup \ldots \cup p_{k}>t$, and hence by condition $(\rho)$ it follows that $t \cap\left(p_{i} \cup p_{j}\right)>a$. Since every chain in $p_{i} \cup p_{j} / a$ has length at most

(2) This definition of point lattice differs somewhat from the usual one. 
two, we must have $t \cap\left(p_{i} \cup p_{j}\right)>a$. But since $p_{i} \cup\left(t \cap\left(p_{i} \cup p_{j}\right)\right)=p_{i} \cup p_{j} \geqq p_{j}$, it follows that $t_{1} \geq t \cap\left(p_{i} \cup p_{j}\right)$, contrary to the definition of $t_{1}$. Hence $c=p_{1} \cup \ldots \cup p_{k}$, and Theorem 3.2 follows.

Theorems 3.1 and 3.2 together give one of the main results of [1] mentioned in the introduction: every element of a semimodular, compactly generated, atomic lattice $L$ has replaceable (irredundant) decompositions if and only if $L$ is locally modular.

A compactly generated atomic lattice $L$ has unique irredundant decompositions if and only if $L$ is locally distributive, and hence $L$ has unique decompositions if and only if $u_{a} / a$ has unique decompositions for each $a \in L$. Similarly, if $L$ is a semimodular, compactly generated, atomic lattice, then $L$ has replaceable decompositions if and only if every $u_{a} / a$ has this property. Therefore the uniqueness of decompositions in a general lattice and the replacement property in a semimodular lattice are "local" properties in the sense that they are determined by the sublattices $u_{a} / a$.

In passing to the question of replaceable decompositions in a general compactly generated atomic lattice $L$ a different situation is encountered. If $L$ is finite dimensional, then it follows almost trivially that $L$ satisfies condition $(\rho)$ if and only if $u_{a} / a$ satisfies $(\rho)$ for each $a \in L$. If finite dimensionality is dropped, however, then $L$ need not satisfy $(\rho)$ even though $u_{a} / a$ satisfies (o) for every $a \in L$. Thus, unlike that of unique decompositions, the property of replaceable decompositions is fundamentally different in the general case than in the finite dimensional case. The following example illustrates this.

Let $L_{1}$ be the lattice comprised of two infinite chains $a_{1}<a_{2}<\ldots$ $<a_{i}<\cdots$ and $b_{1}<b_{2}<\cdots<b_{i}<\cdots$, such that $b_{i}>a_{i}$, and such that the sublattice $b_{i+1} / a_{i}$ is isomorphic with the lattice of Figure 1 for each $i=1,2, \cdots$. Notice that the lattice of Figure 1 has the property that $p_{i, 1} \cup a_{i+1}=b_{i+1}$, and $p_{i, k} \cup a_{i+1}=p_{i+1, k-1}$ for $k \geqq 2$. Hence in $L_{1}$, for every $i$ and $k$ we have $p_{i, k} \cup a_{i+k}=b_{i+k}$. Now let $L$ be the lattice of ideals of $L_{1}$. Every nonprincipal ideal of $L_{1}$ necessarily contains the ideal( $\left.{ }^{3}\right) A=\left(a_{1}, a_{2}, a_{3}, \cdots\right)$. Suppose $B$ is an ideal of $L_{1}$ with $B>A$. Then $B$ must contain the element $p_{i, k}$ for some $i$ and $k$. Thus $B$ contains $p_{i, k} \cup a_{i+k}=b_{i+k}$, and hence $B \geqq\left(b_{1}, b_{2}, b_{3}, \cdots\right)=L_{1}$. Hence $A$ and $L_{1}$ are the only nonprincipal ideals of $L_{1}$, whence it follows that $L$ is compactly generated and atomic. Now the only ideals $X$ covered by more than two elements of $L$ are the ideals $\left(a_{i}\right)$. Thus if $X \neq\left(a_{i}\right)$, then clearly $u_{X} / X$ satisfies $(\rho)$. On the other hand, $u_{\left(a_{i}\right)} /\left(a_{i}\right)$ $\cong b_{i+1} / a_{i}$, and it is apparent from Figure 1 that $u_{\left(a_{i}\right)} /\left(a_{i}\right)$ also satisfies $(\rho)$ for each $i$. However, $L$ does not satisfy $(\rho)$, since $u_{\left(b_{1}\right) \cup A / A}=L_{1}>A$ and $u_{\left(b_{1}\right) /\left(b_{1}\right) \cap A}$ $=u_{\left(b_{1}\right) /\left(a_{1}\right)}=\left(b_{1}\right)$ does not cover $\left(a_{1}\right)$.

We may also consider the stronger replacement property described in [1, Theorem 4.6]. With this in mind, let us define an element $a$ in a compactly generated atomic lattice $L$ to have doubly replaceable irredundant de-

$\left.{ }^{3}\right)\left(x_{1}, x_{2}, \cdots\right)$ denotes the ideal generated by the set $\left\{x_{1}, x_{2}, \cdots\right\}$. 


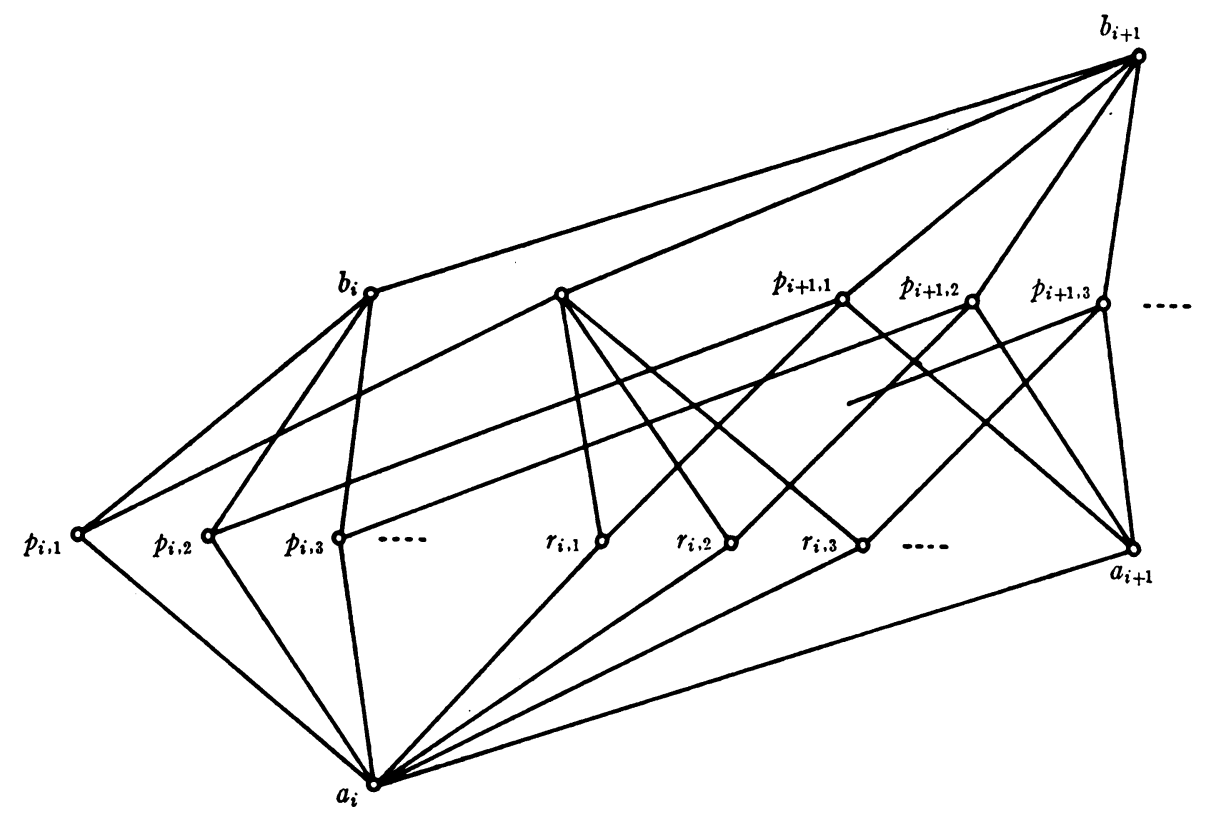

FIGURE 1

compositions if for every pair of irredundant decompositions $a=\cap Q=\cap Q^{\prime}$ and each $q \in Q$ there exists $q^{\prime} \in Q^{\prime}$ such that $a=q^{\prime} \cap \cap(Q-q)=q \cap \cap\left(Q^{\prime}-q^{\prime}\right)$.

THEOREM 3.3. Every element of a semimodular, compactly generated, atomic lattice $L$ has doubly replaceable irredundant decompositions if and only if $u_{a} / a$ is a direct product of finite dimensional modular lattices for every $a \in L$.

Proof. The notation used in this proof is that of $[1, \S 4]$.

If $u_{a} / a$ is a direct product of finite dimensional modular lattices, then it is easily checked that $h_{Q}=u_{a}$ for every irredundant decomposition $a=\cap Q$. Hence the sufficiency follows from [1, Theorem 4.6]( $\left.{ }^{4}\right)$.

The proof of the necessity depends on the following well known lemma essentially due to Frink [2].

LeMma 3.3. If $K$ is a complete, atomic, complemented, modular lattice with a null element $z$, then $K$ is a direct product of quotient sublattices $e_{\alpha} / z$, where each sublattice $e_{\alpha} / z$ has the property that the join of any pair of distinct elements covering $z$ contains a third distinct element covering $z$.

Suppose every element of a semimodular, compactly generated, atomic lattice $L$ has doubly replaceable decompositions. Then it follows that $L$ is locally modular. Let $a \in L$. Since $u_{a} / a$ is complemented by [1, Lemma 4.1], it follows by the preceding lemma that $u_{a} / a$ is a direct product of sublattices

(4) Even though [1, Theorem 4.6] and its corollary are stated for modular lattices, they clearly hold in the locally modular case. 
$e_{\alpha} / a$ each of which has the property expressed in the lemma. Suppose one of the sublattices, say $e_{\beta} / a$, is not finite dimensional. Then $e_{\beta} / a$ contains an infinite independent set $J$ of elements covering $a$. We may assume that $J$ is a maximal independent subset of $\left\{p>a \mid p \leqq e_{\beta}\right\}$, and hence $U J=e_{\beta}$. Pick $p_{0} \in J$. Then for each $p \in J-p_{0}$ there is an element $p>a$ such that $p_{0} \cup p \geqq \bar{p}$ and $p \neq p_{0}, p$. Define

$$
s_{p}=\not \cup \cup\left(J-\left\{p_{0}, p\right\}\right)
$$

for each $p \in J-p_{0}$, and set $S=\left\{s_{p} \mid p \in J-p_{0}\right\}$. Then $e_{\beta}>s_{p}$ and $s_{p} \geq p_{0}, p$ for each $p \in J-p_{0}$. Suppose $\cap S>a$. Then $\cap S \geqq p^{\prime}$ for some $p^{\prime}>a$. Since $U J$ $=e_{\beta} \geqq p^{\prime}$, there is a minimal finite subset $J^{\prime} \subseteq J$ such that $U J^{\prime} \geqq p^{\prime}$. If $p_{0} \in J^{\prime}$, then it follows from semimodularity and the minimality of $J^{\prime}$ that $p^{\prime} \leqq U J^{\prime}$ implies $p_{0} \leqq p^{\prime} \cup \cup\left(J^{\prime}-p_{0}\right)$. Since $J$ is infinite, there exists $p \in J-J^{\prime}$, whence

$$
p_{0} \leqq p^{\prime} \cup \cup\left(J^{\prime}-p_{0}\right) \leqq p^{\prime} \cup \cup\left(J-\left\{p_{0}, p\right\}\right) \leqq s_{p},
$$

a contradiction. If $p_{0} \in J^{\prime}$, then with $p \in J^{\prime}$ we again have

$$
p \leqq p^{\prime} \cup \cup\left(J^{\prime}-p\right) \leqq p^{\prime} \cup \cup\left(J-\left\{p_{0}, p\right\}\right) \leqq s_{p},
$$

a contradiction. Hence $\cap S=a$, and clearly $\cap\left(S-s_{p}\right) \geqq p$ for each $p \in J-p_{0}$.

Now consider $h_{S}$. Since $J$ is independent, $h_{S}=U\left(J-p_{0}\right) \geq p_{0}$, and hence $h_{S} \neq e_{\beta}$. Thus by the corollary to $[1$, Theorem 4.6] $a$ cannot have doubly replaceable decompositions in the sublattice $e_{\beta} / a$. But $u_{a} / a$ is a direct product of $e_{\beta} / a$ and $\left(\bigcup_{\alpha \neq \beta} e_{\alpha}\right) / a$, whence it follows that $a$ cannot have doubly replaceable irredundant decompositions in $L$, contrary to assumption. Thus each $e_{\alpha} / a$ is finite dimensional, and the proof is complete.

\section{REFERENCES}

1. R. P. Dilworth and P. Crawley, Decomposition theory for lattices without chain conditions, Trans. Amer. Math. Soc. vol. 97 (1960) pp. 1-22.

2. O. Frink, Complemented modular lattices and projective spaces of infinite dimension, Trans. Amer. Math. Soc. vol. 60 (1946) pp. 452-467.

California Institute of Technology,

Pasadena, California

UNIVERSITY OF WASHINGTON,

Seattre, Washington 